\title{
OH/IR STARS NEAR THE GALACTIC CENTRE
}

\author{
B. Baud \\ Sterrewacht, Huygens Laboratorium \\ Leiden, The Netherlands.
}

\section{ABSTRACT}

Observations at $1612 \mathrm{MHz}$ around the Galactic Centre have revealed the existence of $43 \mathrm{OH} / \mathrm{IR}$ stars. Sources within $6^{\circ}$ longitude from the Galactic Centre appear to be associated with the central parts of the Galaxy. They have a large velocity dispersion and a mean radial velocity of $0 \mathrm{~km} \mathrm{~s}^{-1}$, indicating that there is no net rotation around the Centre. Within $2^{\circ}$ from the Centre there is a strong relative increase in the number of sources with a velocity separation between the two emission peaks of less than $30 \mathrm{~km} \mathrm{~s}^{-1}$. This is discussed in terms of a possible interaction between the circumstellar shell and the ambient medium.

\section{INTRODUCTION}

In a systematic survey for $\mathrm{OH}$ emission at $1612 \mathrm{MHz}$ of an L-shaped region around the Galactic Centre (Baud et a1. 1977) we have discovered 43 sources which have emission characteristics typical of Type II OH/IR stars: $1612 \mathrm{MHz}$ point source emission at two different velocities, separated over $20-40 \mathrm{~km} \mathrm{~s}^{-1}$, with steep outer edges of the double profile and more gradual inner slopes (see the review by Habing at this Colloquium). The associated stars are considered to be long-period Mira variables. All sources, 38 of which were new discoveries, have been confirmed in August 1977, using the NRAO 43m telescope. The latter observations were made with high velocity resolution $\left(1.2 \mathrm{~km} \mathrm{~s}^{-1}\right)$ in order to accurately obtain the shape and intensity of the line profile.

We argue that the majority of the sources 1 ie within $1 \mathrm{kpc}$ distance from the Galactic Centre (G.C.). Hence intrinsic $1612 \mathrm{MHz}$ luminosities can be derived in a direct way. 
II. RESULTS

1) Distribution of $\mathrm{OH} / \mathrm{IR}$ stars near the Galactic Centre. In Fig. 1 the distribution of $\mathrm{OH} / \mathrm{IR}$ stars in the direction of the G.C. is shown. The region within the continuous lines was surveyed to a 30 sensitivity of $0.7 \mathrm{Jy}$, and a sensitivity of $0.3 \mathrm{Jy}$ was reached in the narrow strip along the galactic plane, enclosed by the dashed lines. The three sources at positive latitudes outside the surveyed regions were previously known.

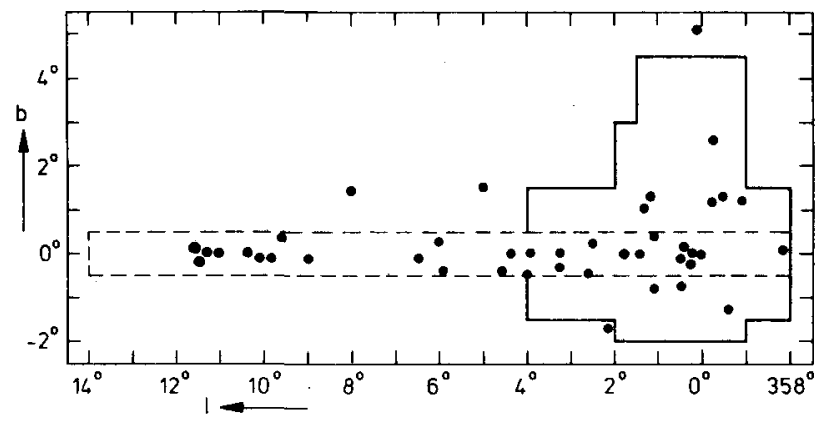

Fig. I Distribution of OH/IR stars near the G.C.. The continuous line shows the area surveyed with a sensitivity of $0.7 \mathrm{Jy}$; the area within the dashed line is surveyed to a sensitivity of $0.3 \mathrm{Jy}$.

There is a modest increase in source density along the plane towards $\ell=0^{\circ}$. However, we may assume that considerably more sources exist within $1^{\circ}$ distance from the G.C. which have not yet been detected because of the intense continuum background in the region and the associated strong and broad oH absorption. Subsequent elaborate observations within 0.5 distance from the G.C. have indeed shown a wealth of new $\mathrm{OH} / \mathrm{IR}$ sources.

The latitude distribution is clearly concentrated to the galactic plane, even when the varying sensitivity has been taken into account. The distribution in latitude near the G.C. is similar to that around $\ell=30^{\circ}$ (Johansson et al, 1977): most sources lie within $1^{\circ}$ distance from the plane. 
2) Radial velocities. The radial velocity distribution of OH/IR stars as a function of galactic longitude is shown in Fig. 2. The curve indicates the maximum radial velocity allowed by circular rotation according to a mass model of the central parts of the Galaxy by Sanders and Lowinger (1972). The distribution is similar to that found for planetary nebulae near the Centre (Minkowski 1965). Although at larger longitudes, the sources seem to follow galactic rotation, for $\ell<6^{\circ}$ there is a marked increase in the number of sources with large negative velocities. The mean radial velocity for all $\mathrm{OH} / \mathrm{IR}$ stars at $l<6^{\circ}$ is about $0 \mathrm{~km} \mathrm{~s}$. This result and the dramatic increase in the radial velocity dispersion at small longitudes to a value of $\sim 120 \mathrm{~km} \mathrm{~s}^{-1}$ indicates that most OH/IR stars within $\ell=6^{\circ}$ are physically associated with the central region of the Galaxy and lie within 1 kpc distance from the Centre.

It should be noted that absorption due to foreground molecular clouds may considerably attenuate the emission of stars that lie behind these molecular clouds and coincide in radial velocity. This can influence the observed radial velocity distribution. Strong, broad absorption is present at negative radial velocities around $\ell=0^{\circ}$ and at positive radial velocities below $+160 \mathrm{~km} \mathrm{~s}^{-1}$ at $\ell \leq 3$. Thus the low velocity sources near the G.C. are probably undersampled. No absorption is visible at $\mathrm{V} z+160 \mathrm{~km} \mathrm{~s}^{-1}$. The lack of sources with high positive radial velocities $\left(\mathrm{V}>+160 \mathrm{~km} \mathrm{~s}^{-1}\right)$ at positive longitudes appears therefore real.

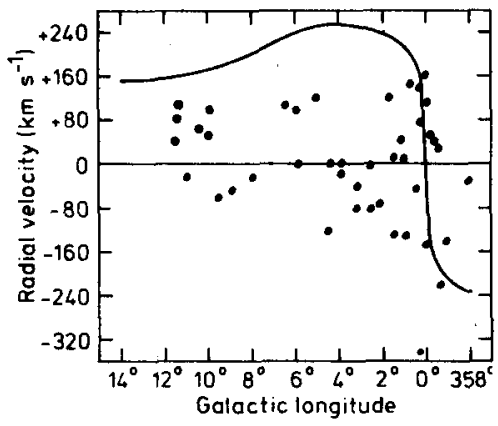

Fig. 2 Radial velocities as a function of longitude. The curve represents the maximum radial velocity for circular orbits around the G.C. according to Sanders and Lowinger (1972). 


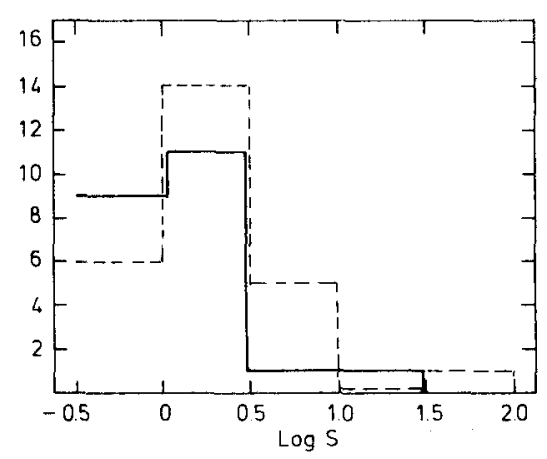

Fig. 3 Luminosity distribution of $1612 \mathrm{MHz}$ emission from OH/IR stars near the G.C. with $|b| \leq 0.5$, for $\ell \geq 6^{\circ}$ (drawn) and $\ell<6^{\circ}$ (dashed). Sources that are separated less than 0.5 longitude from the G.C. have been excluded because of a variable sensitivity of the observations due to increased continuum emission in that region.

3) Emission characteristics of $\mathrm{OH} / \mathrm{IR}$ stars near the Galactic Centre. We have shown that most stars within $\ell<6^{\circ}$ distance are probably physically associated with the Centre and hence are at about the same distance from the Sun. Their observed 1612 MHz luminosities should therefore reflect their intrinsic luminosity distribution. In Fig. 3 two curves have been drawn: the continuous curve represents all stars in the narrow $\left(1^{\circ}\right)$ strip along the plane with $\ell>6^{\circ}$; the dashed line the stars with $358^{\circ}<\ell<6^{\circ}$, excluding all those with $\ell<1^{\circ}$. This is because of the decrease in survey sensitivity mentioned in $\$ 2$. The strong decrease in the dashed curve for $\log \mathrm{S}<0$ is real and not due to observational selection effects. The difference between the two curves reflects an essential difference in the density distribution of sources along the line of sight. The drawn curve is compatible with a uniform density distribution. If there is a maximum along the line of sight a curve similar to the dashed curve results. Its symmetrical shape indicates that the apparent luminosity distribution is not smeared by a large range in distances. It confirms our conclusion that most stars at $\ell<6^{\circ}$ are at the same distance from the Sun. The width of the distribution of $\sim 1.0$ reflects the range in intrinsic luminosities and corresponds to a factor of 10 in intrinsic luminosities. The mean value of $\log \mathrm{S}$ is equal to 0.27 corresponding to a mean peak flux density of $1.9 \mathrm{Jy}$, which is more than four times lower than the value derived from the intrinsic luminosities of OH/IR 
stars at galactic longitudes larger than $18^{\circ}$, as determined by Johansson et al. (1977). This difference cannot be accounted for by the difference in spectral resolution between their observations $(1 \mathrm{kHz})$ and our measurements $(6.2 \mathrm{kHz})$, because the emission features are usually more than $6 \mathrm{kHz}$ wide. They determined a mean intrinsic luminosity in a statistical way, using both radial velocities and observed peak flux densities in order to reduce the ambiguities between near and far kinematical distance. Even if all sources they find are situated at the near kinematical distance - which is unlikely - the difference between the mean intrinsic luminosity they find and the value we derive for $\mathrm{OH} / \mathrm{IR}$ stars near the Galactic Centre would still be more than a factor of three. We therefore conclude that this difference is real and that the OH/IR stars in the central parts of the Galaxy are less luminous at $1612 \mathrm{MHz}$ than those found at large distances ( $7 \mathrm{kpc}$ ) from the nucleus.

The velocity separation $\Delta V$ between the two emission peaks is related to the luminosity of the underlying star and its mass loss rate (see the contribution by Olnon at this colloquium). Knowledge of the distribution in $\Delta V$ may therefore be important in deriving the physical properties of the stars associated with the $\mathrm{OH}$ maser phenomenon. The present data suggest that the distribution of $\Delta V$ very close to the G.C. differs from that elsewhere in the Galaxy. This may be presented in two somewhat different ways. Fig. 4 shows the distribution in $\Delta V$ for three separate longitude intervals. The peak clearly shifts from $\Delta V \approx 32 \mathrm{~km} \mathrm{~s}^{-1}$ at $\ell>2^{\circ}$ to $\Delta V \approx 27 \mathrm{~km} \mathrm{~s}^{-1}$ at $\ell<2^{\circ}$. There is also an indication that the width of the $\Delta V$ distribution close to the Centre is diminished: no stars have been found in that region with $\Delta \mathrm{V}>40 \mathrm{kn} \mathrm{s}^{-1}$. In Fig. 5 we have drawn the galactic longitude distribution of stars with $\Delta \mathrm{V} \geq 30 \mathrm{~km} \mathrm{~s}^{-1}$ (dashed) and $\Delta \mathrm{V}<30 \mathrm{~km} \mathrm{~s}^{-1}$ (drawn). The former distribution is practically constant but there is a strong increase of more than a factor of two in the number of sources with $\Delta V<30 \mathrm{~km} \mathrm{~s}^{-1}$ within $l=2^{\circ}$. The same increase is clearly visible for stars within the strip of $1^{\circ}$ width along the galactic plane, indicating that this is not due to some kind of selection effects introduced by the increased latitude coverage at $\ell<2^{\circ}$.

One can only speculate as to the cause of this shift to lower values of $\Delta V$ in the nuclear region of the Galaxy. If it has the same cause as the lower $1612 \mathrm{MHz}$ luminosity one would expect a relation between $\Delta V$ and peak flux density for the OH/IR stars near the Galactic Centre. In the data presently discussed no such relation has been found. Another possible explanation is the interaction of the expanding shell, in which the masing occurs, with the surrounding medium by sweeping up material in the course of expansion. This may become important in the nucleus of the Calaxy, where the gas density is considerably higher than that found in the disk. Conservation of linear momentum of the expanding shell will result in a deceleration of expansion and hence a decrease in the observed $\Delta V$, proportional to the fraction 


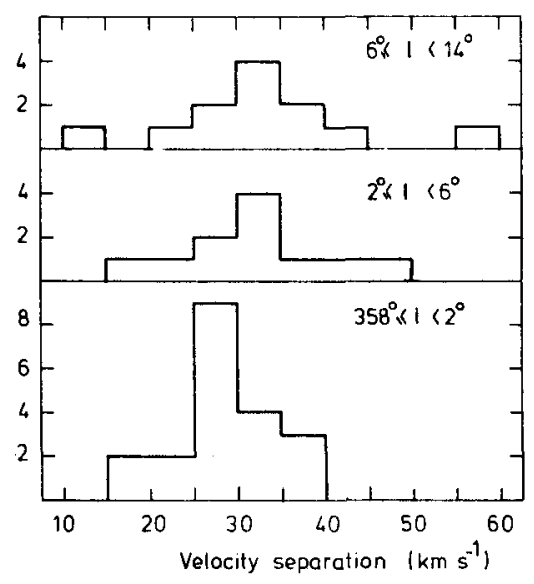

Fig. 4 Distribution of $\Delta V$ for $O H / I R$ sources in three separate longitude intervals.

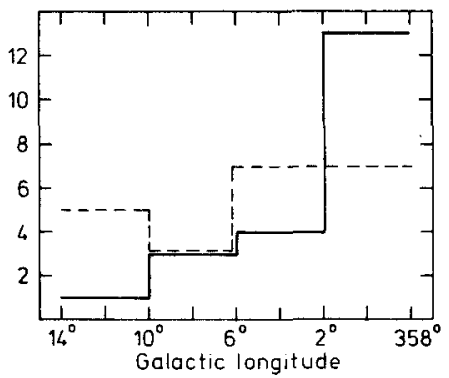

Fig. 5 Longitude distribution of $\mathrm{OH} / \mathrm{IR}$ stars with $\Delta \mathrm{V}<30 \mathrm{~km} \mathrm{~s}^{-1}$ (continuous) and $\Delta \mathrm{V} \geq 30 \mathrm{~km} \mathrm{~s}^{-1}$ (dashed).

of the accreted mass to the total mass of the shell. If a shell has initially a radius of $3 \times 10^{16} \mathrm{~cm}$, a density of $2 \times 10^{4} \mathrm{~cm}^{3}$ (E1itzur et al. 1976) and if it expands into a medium with a density of $1 \mathrm{~cm}^{-3}$, then it has to expand to a size of $5 \times 10^{17} \mathrm{~cm}$ before $i t$ has swept up enough mass to decelerate to the observed lower expansion velocity of $27 \mathrm{~km} \mathrm{~s}^{-1}$. This will occur in a time scale of $10^{4}$ years, 
assuming an expansion velocity of $15 \mathrm{~km} \mathrm{~s}^{-1}$. All $0 \mathrm{H} / \mathrm{IR}$ stars up measured to date with VLBI techniques have emission regions that are at least ten times smaller (Reid et al. 1977), but these stars are situated in the neighbourhood of the Sun, where the density of the interstellar medium is much lower. VLBI experiments on the OH/IR stars discussed here are needed to determine the size of the masing regions.

The large velocity dispersion of OH/IR stars near the Centre suggests that many of these stars travel with large velocities through the ambient medium, which follows more or less circular rotation. As a result a considerable amount of matter may be swept up by the circumstellar she11. Because the interstellar matter is asymmetrically swept up on that side of the shell that lies in front with respect to the direction of motion, the accreting shell will slow down, while the associated star moves on. Assuming that the star moves through an ambient medium of density $1 \mathrm{~cm}^{-3}$ with a velocity of $75 \mathrm{~km} \mathrm{~s}^{-1}$, this asymmetric accretion process results in a considerable slow-down of the shell with respect to the star and will cause them to be separated in less than $10^{4}$ years. Such a time scale is comparable to or somewhat smaller than the time scale of the mass loss phase for Mira variables (Kafatos et al. 1977). If indeed the OH masing phase of such stars in the central parts of the Galaxy is shortened by the above mentioned process then the observed frequency distribution of $\mathrm{OH} / \mathrm{IR}$ stars near the G.C. is only a lower limit and we expect that many more potential OH/IR stars exist which have been stripped of their circumste11ar shell.

\section{REFERENCES}

Baud, B., Habing, H.J., Matthews, H.E., Winnberg, A. 1977, in preparation. Elitzur, M., Goldreich, P., Scoville, H. 1976, Astrophys. J. 205, 384.

Johansson, L.E.B., Andersson, C., Goss, W.M., Winnberg, A. 1977, Astron. and Astrophys. $54,323$.

Kafatos, M., Michalitsanos, A.G., Vardya, M.S. 1977, Astrophys. J. 216, 526.

Minkowski, R. 1965, Stars and Stellar Syst. V, 321.

Reid, M.J., Muhleman, D.0., Moran, J.M. Johnston, K.J., Schwartz, P.R. 1977, Astrophys. J. 214, 60 .

Sanders, R.H., Lowinger, T. 1972, Astron. J. 77, 292.

D I S C US S I O N of paper by BAUD:

SHERHOOD: 1. Simultaneous $O H$ and IR observations of IR sources at the galactic centre is a method of identifying the IR counterpart of the $\mathrm{OH}$ source.

2. Our group has found an IR source near OH $0.33-0.19$ but, as I showed, the IR energy distribution of OH/LR sources is not unique. Further information is required: either simultaneous $O H$ and IR observations (unique identification) or spectroscopic identification (probably not unique). 
MERRILL: The OH/IR stars can be classified as Mira variables or $M$ supergiants by moderate resolution spectra of the $2-4 \mu$ region where the $\mathrm{CO}-\mathrm{H}_{2} \mathrm{O}$ absorption in $\mathrm{coOl}$ stellar photospheres $\mathrm{can}$ be identified. Such studies are currently being pursued by a number of observers.

BAUD: If such absorption is uniquely associated with OH/IR stars, the identification of IR points sources with $O H$ maser sources will become much easier in the central regions of the Galaxy, than looking for simultaneous variability in the OH and IR emission, which now seems to be the only other safe way for identification.

HABING : Radio Astronomers are also plagued by interstellar extinction. Molecular clouds in the centre give significant absorption in the $\mathrm{OH}$ line at $1612 \mathrm{MHz}$, and they dim the signals from $\mathrm{OH} / \mathrm{IR}$ stars behind the clouds noticeably.

GEYER: I have an optical blink survey from R- and I-Schmidt plates of very red stars with $R-F \geq 2^{m}$ covering more than 10 years at my disposal. There is a quite good correlation with your presented data. Unfortunately, I have not published this large amount of data.

BAUD: Many OH/IR stars are heavily reddened, as Sherwood and Merrill have shown. They are probably not visible on such plates. Observations by Wynn-Williams et al. in various wavelength regions longward of $2 \mu$ of the Galactic centre, do not show any point sources, that coincide with the OH/IR stars that we have found. Identification of the stars is probably best done on the basis of simultaneous variation of the OH and IR emission, as is seen in many nearby OH/IR stars. 